\title{
New methodology for the heat flow prediction for different construction materials
}

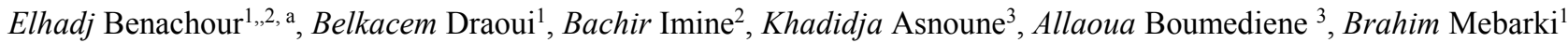 \\ ${ }^{1}$ Laboratory of Energy in Arid Regions, University of Tahri Mohamed - Bechar, P.O. P 417. 08000, Road \\ Kenedza , Bechar, Algeria \\ ${ }^{2}$ Department of Mechanical Engineering, University of Sciences and Technology of Oran -Mohamed Boudiaf, \\ P.O.1505 El-Mnaouar, Oran, Algeria \\ ${ }^{3}$ Laboratory of Smart Grids and Renewable Energies, TAHRI Mohammed, University of Bechar, BP. 417 \\ Bechar (08000), Algeria
}

\begin{abstract}
Among the ways of energy transfer by conduction is that of molecular interaction, in which the greater motion of a molecule at a higher energy level (temperature) imparts energy to adjacent molecules at lower energy levels. This type of transfer is present, to some degree, in all systems in which a temperature gradient exists and in which molecules of a solid, liquid, or gas are present. So, in heat transfer, the thermal conductivity of a substance is an intensive property that indicates its ability to conduct heat In particular in the building sector. The thermal flux is often measured with a mathematical analysis but for the same material, on the other hand the estimate will be disruptive and sometimes very difficult when the material changes. In this paper, a single equation for predicting heat flux of different materials is given.
\end{abstract}

\section{Introduction}

According to the Second Law of Thermodynamics, heat transfer is only possible in the direction from higher temperatures to a lower one. It becomes zero if temperatures are equal. The heat loss through an envelope should therefore be proportional to the difference Temp (inside) - Temp (outside), or to a positive power of it for small differences. For a simple formula, a linear dependence on temperature difference is sufficient. Accepting further that heat loss grows linearly with the surface. Knowing how heat "flows" inside a structure is significant for designers to make sure that the structure will perform its intended heat conduction function properly [1]. Several works are interested in the conduction heat transfer in several materials and several domains [2-11], and other works are interested in convective heat transfer and conduction convection coupling [12-13]. Irrespective of the mode of heat transfer, the researcher is interested in the energy flow through which is diffused by this building material among the most important physical and thermal properties there is thermal conductivity. In this work, we will vary the conductivity thermal analysis of four different materials: wood, concrete, brick and granite, and mathematical calculation of the thermal flux to find a point cloud that helps us to use Lagrange's polynomial interpolation method to develop a only correlation to estimate the heat flux diffused by one of these four materials proposed at all times.

\section{Geometric configuration}
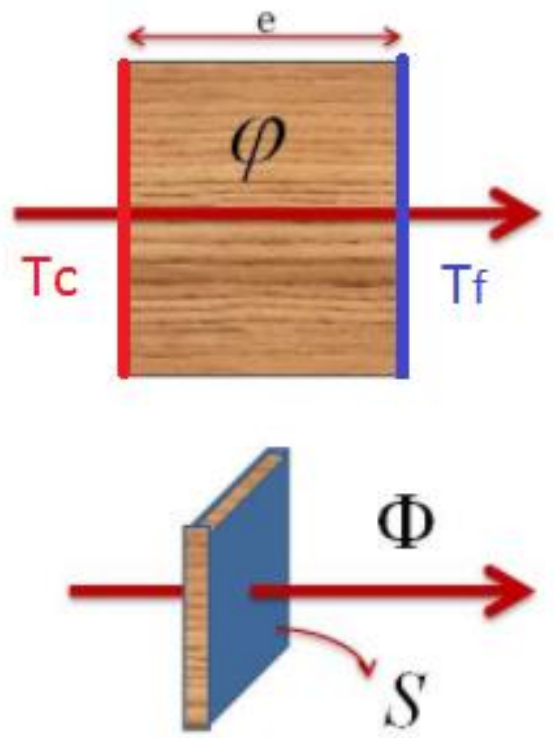

Fig. 1. Schematic of the studied configuration

To simplify the problem, assume that:

$>$ Heat transfer is in the direction of decreasing temperature (basis for minus sign).

\footnotetext{
$\bar{a}$ Corresponding author: benachour_elhadj@yahoo.fr
} 
Direction of heat transfer is perpendicular to lines of constant temperature (isotherms).

$>$ Heat flux vector may be resolved into orthogonal components

$>$ For transient conduction, heat equation is first order in time, requiring specification of an initial temperature distribution

\section{Objectives}

We can express our aim for this study in the following points:

- $\quad$ study of heat transfer by conduction in different types of material

- Studied the effect of thermal conductivity on thermal flux and conduction heat transfer in different types of material

- Prediction of the Heat flow for different construction materials.

\section{Mathematical model}

The differential form of the Fourier's Law of heat conduction is

$$
\bar{q}=-\lambda \nabla T
$$

where $\bar{q}$ is the local heat flux, $\lambda$ is the thermal conductivity, and $\mathrm{T}$ is the local temperature. The Fourier's law is very simple in mathematics and has been widely used in engineering applications and in most scientific research at life scale, even though it is only an empirical relationship.

Experience leads us to believe that as e increases $\Phi$ should get smaller. All of these lead to the generalization (made by Fourier in 1807).

A more useful quantity to work with is the heat transfer per unit area, defined as:

$$
\Phi=q \cdot S
$$

where:

$$
q=-\lambda \frac{d T}{d x}
$$

the quantity $\mathrm{q}$ is called the heat flux and its units are $\mathrm{W} / \mathrm{m}^{2}$. The expression in (2) can be written in terms of heat flux as:

$$
\Phi=-\lambda \frac{d T}{d x} \cdot S
$$

for one-dimensional heat conduction (temperature depending on one variable only), we can devise a basic description of the process.

The heat transfer rate in at the left (at $\mathrm{x}$ ) is:

$$
\Phi(x)=-\lambda\left(S \frac{d T}{d x}\right)_{x}
$$

the heat transfer rate on the right is

$$
\Phi(x+d x)=\left(\Phi(x)+\left.\frac{d \Phi}{d x}\right|_{x} d x+\ldots \ldots \ldots .\right.
$$

using the conditions on the overall heat flow and taking the limit as dx approaches zero we obtain

$$
\frac{d \Phi(x)}{d x}=0
$$

or

$$
\frac{d}{d x}\left(\lambda \frac{d T}{d x} \cdot S\right)=0
$$

if $\lambda$ is constant (i.e. if the properties independent of temperature), this reduces to

$$
\frac{d}{d x}\left(\frac{d T}{d x} \cdot S\right)=0
$$

or (using the chain rule)

$$
\frac{d^{2} T}{d x^{2}}+\left(\frac{1}{S} \frac{d S}{d x}\right) \frac{d T}{d x}=0
$$

equations (9) or (10) describe the temperature field for quasi-one-dimensional steady state (no time dependence) heat transfer. We now apply this to our example.

For this configuration (Figure1), the area is not a function of $\mathrm{x}$, i.e. $\mathrm{S}=$ constant. Equation (10) thus became

$$
\begin{gathered}
\frac{d^{2} T}{d x^{2}}=0 \\
\frac{d T}{d x}=A \\
T(x)=A(x)+B
\end{gathered}
$$

equation (13) is an expression for the temperature field where A and B are constants of integration. For a second order equation, such as (11), we need two boundary conditions to determine $\mathrm{A}$ and $\mathrm{B}$. One such set of boundary conditions can be the specification of the temperatures at both sides of the slab as shown in Figure, say $\mathrm{T}(0)=\mathrm{Tc} ; \mathrm{T}(\mathrm{e})=\mathrm{Tf}$.

The condition $\mathrm{T}(0)=\mathrm{Tc}$ implies that $\mathrm{b}=\mathrm{Tc}$. The condition $\mathrm{Tf}=\mathrm{T}(\mathrm{e})$ implies that $\mathrm{Tf}=\mathrm{A}$ e $+\mathrm{Tc}$, or

$$
\begin{gathered}
A=\frac{T f-T c}{e} \\
T(x)=A(x)+B
\end{gathered}
$$

with these expressions for $\mathrm{a}$ and $\mathrm{b}$ the temperature distribution can be written as 


$$
T(x)=T c+\left(\frac{T f-T c}{e}\right) x
$$

this linear variation in temperature is shown in Figure 2 for a situation in which $\mathrm{Tc}>\mathrm{Tf}$.

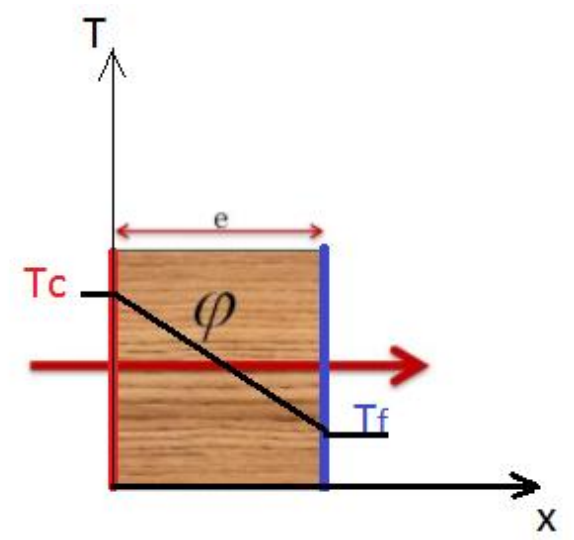

Fig. 2. Temperature distribution through a slab

The heat flux $\mathrm{q}$ is also of interest. This is given by

$$
q=-\lambda \frac{d T}{d x}=-\lambda \frac{(T f-T c)}{e}=\text { constant }
$$

There is an electrical analogy with conduction heat transfer that can be exploited in problem solving. The analog of $\mathrm{Q}$ is current, and the analog of the temperature difference, Tc - Tf, is voltage difference. From this perspective the slab is a pure resistance to heat transfer and we can define

$$
\dot{\Phi}=\frac{(T f-T c)}{R}=\frac{\Delta T}{R}
$$

where the thermal resistance is given by:

$$
R=\frac{e}{\lambda S}
$$

\section{Procedure of Simulation}

Of course the only mode of heat transfer is conduction but the resistance of the different materials change despite us being interested at the moment when the temperature gradients are equal, due to change in thermal conductivity.

Then, firstly, the thermal flux of each material is calculated mathematically to obtain a cloud of points. then the polynomial interpolation technique will be used to develop a correlation that aggregates all thermal transfer diffusion data in all materials regardless of thermal conductivity.

\section{Mathematical model for The Lagrange polynomial Interpolation Method}

The Lagrange interpolating polynomial is the polynomial of degree $\leq(n-1)$ that passes through the $\mathrm{n}$ points $(\mathrm{x} 1, \mathrm{y} 1=\mathrm{f}(\mathrm{x} 1)),(\mathrm{x} 2, \mathrm{y} 2=\mathrm{f}(\mathrm{x} 2)), \ldots,(\mathrm{xn}$, $\mathrm{yn}=\mathrm{f}(\mathrm{xn}))$, the interpolation polynomial in the Lagrange form is a linear combination [14] and is given by :

$$
P_{n}(x)=\sum_{i=0}^{n} y_{i} \quad L_{i}(x)
$$

for Lagrange basis polynomials

$$
L_{i}(x)=\frac{\prod_{\substack{j=0 \\ j \neq i}}^{n}\left(x-x_{j}\right)}{\prod_{\substack{j=0 \\ j \neq i}}^{n}\left(x_{i}-x_{j}\right)}=\prod_{\substack{j=0 \\ j \neq i}}^{n} \frac{\left(x-x_{j}\right)}{\left(x_{i}-x_{j}\right)}
$$

where

$$
\begin{cases}L_{i}\left(x_{j}\right)=0 & \text { if } \quad i \neq j \\ L_{i}\left(x_{j}\right)=1 & \text { if } \quad i=j\end{cases}
$$

\section{Results and Discussion}

\subsection{The polynomial interpolation points}

We wish to find the polynomial interpolating the points

Table 1. The sets of polynomial interpolation points.

\begin{tabular}{|c|c|c|c|c|}
\hline & Wood & Concrete & Brick & Granite \\
\hline$\lambda$ & 0.17 & 1.75 & 1.31 & 0.12 \\
\hline$\Phi$ & 19.55 & 201.25 & 150.65 & 13.8 \\
\hline
\end{tabular}

where, the points of mathematical method.

$\Phi(\lambda)$ are obtained by the If $(0.12,13.8),(0.17,19.55), \quad(1.31,150.65)$, $(1.75,201.25)$, are given data points, then the cubic polynomial passing through these points can be expressed as,

$$
P_{3}(x)=y_{0} l_{0}+y_{1} l_{1}+y_{2} l_{2}+y_{3} l_{3}
$$

We would have the four basis polynomials

$$
\begin{aligned}
& l_{0}=\frac{(x-0.17)(x-1.31)(x-1.75)}{(0.12-0.17)(0.12-1.31)(0.12-1.75)} \\
& l_{1}=\frac{(x-0.12)(x-1.31)(x-1.75)}{(0.17-0.12)(0.17-1.31)(0.17-1.75)} \\
& l_{2}=\frac{(x-0.12)(x-1.17)(x-1.75)}{(1.31-0.12)(1.31-1.17)(1.31-1.75)} \\
& l_{3}=\frac{(x-0.12)(x-0.17)(x-1.31)}{(1.75-0.12)(1.75-0.17)(1.75-1.31)}
\end{aligned}
$$

We can see that each term is of degree three, so the entire sum has degree at most three.

The polynomial $\mathrm{P}(\mathrm{x})$ given by the above formula is called Lagrange's interpolating polynomial and the functions Eq. (23), Eq. (24), Eq (25), Eq.( 26) are called Lagrange's interpolating basis functions. 


\subsection{Heat flow correlation}

It should be noted that the numerical result given by the equation Eq.(20). Remarkably similar to the estimate of the Heat flow by the Lagrange interpolation method for each material which her thermal conductivity between $[0.12,1.75]$.

$$
\begin{gathered}
P_{3}(x)=y_{0} l_{0}+y_{1} l_{1}+y_{2} l_{2}+y_{3} l_{3} \\
P_{3}(x)=0.12 l_{0}+0.17 l_{1}+1.31 l_{2}+1.75 l_{3}
\end{gathered}
$$

Therefore, we can write,

$\Phi(x)=-0.0126 \quad x^{3}-3.565 .10^{-3} x^{2}+114.28 x-0.127$

So, since the variable is the thermal conductivity, finally we can write

$$
\Phi(\lambda)=-0.0126 \quad \lambda^{3}-3.565 .10^{-3} \lambda^{2}+114.28 \lambda-0.127
$$

\section{Comparison and validation of equation}

Table 2. The errors estimate.

\begin{tabular}{|c|c|c|c|c|}
\hline $\begin{array}{c}\text { The } \\
\text { material }\end{array}$ & $\lambda$ & $\begin{array}{c}\Phi(\lambda) \\
\text { The } \\
\text { mathematically } \\
\text { obtained result } \\
\text { (Eq 18) } \\
\text { (exact } \\
\text { solution) }\end{array}$ & $\begin{array}{c}\Phi(\lambda) \\
\text { The results } \\
\text { obtained by } \\
\text { our equation } \\
\text { (Eq 30) } \\
\text { approximate } \\
\text { solution) }\end{array}$ & $\begin{array}{c}\text { Relative } \\
\text { error }\end{array}$ \\
\hline Granite & 0.12 & 13.80 & 13.5865269 & $1.5 \%$ \\
\hline Wood & 0.17 & 19.55 & 19.3004341 & $1.2 \%$ \\
\hline Brick & 1.31 & 150.65 & 149.545349 & $0.73 \%$ \\
\hline Concrete & 1.75 & 201.25 & 199.784546 & $0.73 \%$ \\
\hline
\end{tabular}

From this comparison, it is very clear that the error is very small for any proposed material that it grows and shows the effectiveness of this developed formula.

\section{Methodology testing}

Our model comparison has demonstrated that different structures material can be useful in evaluating heat flow transmission In any chosen material with this equation (see fig.3)

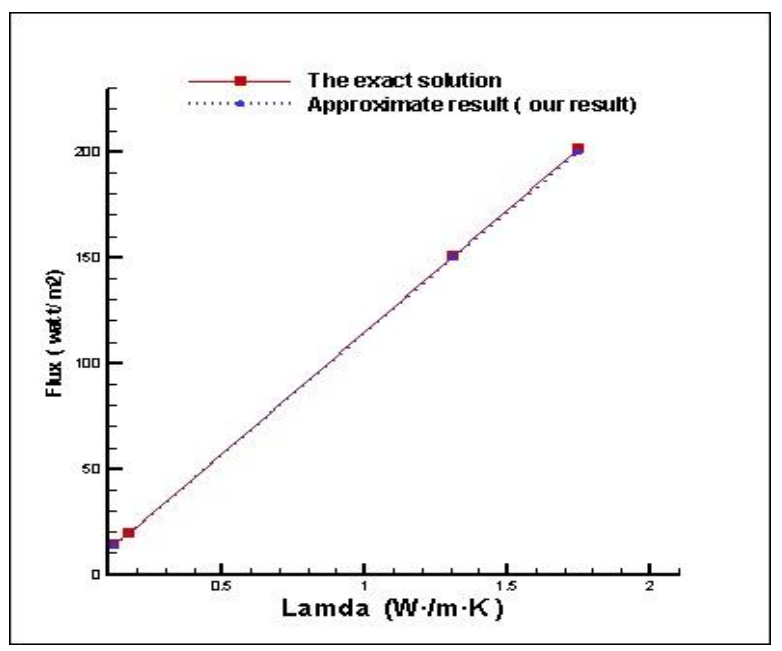

Fig. 3. Comparative study between the exact and approximate solution to estimate the heat flux for different materials

\section{Conclusion}

Knowing how heat "flows" inside a structure is significant for designers to make sure that the structure will perform its intended heat conduction function properly. This paper describes the principle and the process of defining an optimal topology for heat conduction.

Predictive simple correlation (Eq 30) was developed to estimate the Heat flow by the Lagrange interpolation method for each material: wood, concrete, brick and granite And even for all materials that have their thermal conductivity which varies between $[0.12,1.75] \mathrm{W} / \mathrm{m} \cdot \mathrm{K}$. Due to their simplicity and reliability it is very straightforward to use in practice.

\section{Acknowledgment}

This work supported by the Research program sponsored by ENERGARID laboratory, the Faculty of Science and Technology, Tahri Mohamed University, Bechar, Algeria.

I would like to thank all reviewers for taking the time and energy to review our work. 


\section{References}

1. Baotong Li, Jun Hong, Xiangyang Tian, (2016) Generating optimal topologies for heat conduction by heat flow paths identification, International Communications in Heat and Mass Transfer, Volume 75, July 2016, Pages 177-182.

2. Marcin Zych, Robert Hanus, Pavel Vlasak ,Leszek Petryka and Marek Jaszczur, (2016 ). Application of nuclear techniques in two-phase liquid-solid particles hydrotransport investigations, EPJ Web of Conferences 114- 02145 (2016).

3. Arkadi Berezovski,(2016) On the influence of microstructure on heat conduction in solids, International Journal of Heat and Mass Transfer, Vo lume 103, Pages 516-520.

4. G. Medvedev,(2016) High-temperature heating of liquid in a round tube in terms of axial heat conduction, EPJ Web of Conferences 110- 01039 (2016).

5. Vyacheslav I. Maksimov, Tatiana A. Nagornova and Viktor P. Glazyrin, (2016) Conjugate Heat Transfer in a Closed Volume with the Local Heat Sources and Non-Uniform Heat Dissipation on the Boundaries of Heat Conducting Walls, EPJ Web of Conferences 110- 01038 (2016 ).

6. Mustafa Turkyilmazoglu,(2015) Exact Multiple Solutions for the Slip Flow and Heat Transfer in a Converging Channel, Journal of Heat Transfer October 01, 2015 | Volume 137, Issue 10

7. Syed Ashraf Ali and Sandip Mazumder,(2015) Phonon Heat Conduction in Multidimensional Heterostructures: Predictions Using the Boltzmann Transport Equation, J. Heat Transfer 137(10), 102401.

8. J. Ordóñez-Miranda and J. J. Alvarado-Gil, (2011) Effective Thermal Properties of Layered Systems Under the Parabolic and Hyperbolic Heat Conduction Models Using Pulsed Heat Sources, Journal of Heat Transfer, September, 2011 | Volume 133 | Issue 9

9. N. A. Roberts and D. G. Walker, (2011) Computational Study of Thermal Rectification From Nanostructured Interfaces, , Journal of Heat Transfer, September, 2011 | Volume 133 | Issue 9

10. Jing Fan and Liqiu Wang,(2011) Erratum: "Review of Heat Conduction in Nanofluids"'Journal of Heat Transfer, 2011, 133(4), p. 040801].

11. Moran wang, bin-yang cao , zeng-yuan guo,(2010) general heat conduction equations based on the thermomass theory, frontiers in heat and mass transfer (FHMT), 1, 013004 (2010), global digital central issn: 2151-8629.

12. Elhadj Benachour, Belkacem Draoui, Bachir Imine, Mohammed Hasnat and Lakhdar Rahmani(2016), Effect of the insulation by the mud on the convection in building in the Sahara of Algeria case of Bechar City, EPJ Web of Conferences 11402003(2016).
13. Amina Hammadou, Abdelkrim Missoum, Elmir Mohamed and Belkacem Draoui

The effect of the Saharan climate on the indoor environment of a station of electric energy transformation $\mathrm{MV} / \mathrm{LV}$

EPJ Web of Conferences 143, 02032 (2017).

14. Elhadj Benachour, Belkacem Draoui, Bachir Imine, Khadidja Asnoune and Elmir Mohamed (2017), New methodology for the walls design in buildings by numerical simulation of the thermal convection, EPJ Web of Conferences 143, 02007 (2017) 